\title{
CEP-11004, an inhibitor of the SAPK/JNK pathway, reduces TNF- $\alpha$ release from lipopolysaccharide-treated cells and mice
}

\author{
John R. Ciallella ${ }^{\mathrm{a}, *}$, Michael Saporito ${ }^{\mathrm{a}}$, Soren Lund ${ }^{\mathrm{b}}$, Marcel Leist ${ }^{\mathrm{b}}$, Henrik Hasseldam ${ }^{\mathrm{b}}$, \\ Natalie McGann ${ }^{\mathrm{a}}$, Charles S. Smith ${ }^{\mathrm{a}}$, Donna Bozyczko-Coyne ${ }^{\mathrm{a}}$, Dorothy G. Flood ${ }^{\mathrm{a}}$ \\ ${ }^{a}$ Cephalon, Inc., 145 Brandywine Parkway, West Chester, PA 19380, United States \\ ${ }^{\mathrm{b}}$ H. Lundbeck A/S, Valby DK-2500, Denmark
}

\begin{abstract}
CEP 11004, a mixed lineage kinase (MLK) inhibitor, was examined for its effects on tumor necrosis factor alpha (TNF $\alpha$ ) production in human THP 1 monocytes, mouse BV 2 microglia, and C57Bl/6 mice. CEP 11004 inhibited TNF $\alpha$ secretion up to 90\% in THP 1 cells incubated with $3 \mu \mathrm{g} / \mathrm{ml}$ lipopolysaccharide, with an $\mathrm{IC}_{50}$ of $137 \pm 14 \mathrm{nM}$. CEP 11004 also inhibited TNF $\alpha$ production in lipopolysaccharide stimulated microglial cells, but did not inhibit the initial increase in TNF $\alpha$ mRNA expression as measured by real time polymerase chain reaction (PCR). The mitogen activated protein kinases (MAPKs) phospho c jun N terminal kinase (JNK), phospho p38, and phospho MAPK kinase 4 (MKK4) levels were increased in THP 1 cells following lipopolysaccharide treatment, and were reduced by CEP 11004 treatment. For in vivo studies, CEP 11004 was injected $2 \mathrm{~h}$ prior to lipopolysaccharide $(20 \mathrm{mg} / \mathrm{kg})$ administration. CEP 11004 significantly inhibited TNF $\alpha$ production at doses of $110 \mathrm{mg} / \mathrm{kg}$ as measured by enzyme linked immunosorbent assay (ELISA). These results suggest that MLK blockade may be useful in inhibiting pro inflammatory cytokine production in a wide range of diseases.
\end{abstract}

(C) 2005 Elsevier B.V. All rights reserved.

Keywords: Mixed lineage kinase; Tumor necrosis factor-alpha; MAP kinase inhibition

\section{Introduction}

CEP-11004 (Fig. 1) is an indolocarbazole analogue derived from the natural product $\mathrm{K} 252 \mathrm{a}$ and is characterized by its ability to inhibit mixed lineage kinases (MLKs). It inhibits MLK1, MLK2, and MLK3 with $\mathrm{IC}_{50}$ values of 45, 31, and $89 \mathrm{nM}$, respectively (Murakata et al., 2002). CEP11004 was shown to inhibit activation of the mitogenactivated protein kinase (MAPK) c-jun $\mathrm{N}$-terminal kinase (JNK) and to exhibit dose-dependent rescue of rat PC12 cells, human SH-SY5Y cells, and primary rat cortical neurons treated with apoptosis-inducing reagents in vitro. In NGF-deprived sympathetic neurons, CEP-11004 inhibited activation of the JNK pathway (Wang et al., 2005). In vivo studies indicated that administration of CEP-

\footnotetext{
* Corresponding author. Tel.: +1 610738 6186; fax: +1 6107386643 . E-mail address: jciallel@cephalon.com (J.R. Ciallella).
}

11004 inhibited the 1-methyl-4 phenyl-1,2,3,6-tetrahydropyridine (MPTP)-mediated increase in phosphorylated MAPK kinase 4 (MKK4) and attenuated the loss of striatal dopaminergic terminals in mice (Murakata et al., 2002).

The MLKs are a family of serine/threonine kinases that are part of the upstream signaling cascade that activates the MAPKs JNK and p38. The MLKs phosphorylate and activate MKKs, which then activate JNK and p38 (for review, see Gallo and Johnson, 2002). JNK, also called stress-activated protein kinase (SAPK), represents a family of proteins that are key effectors in signal transduction pathways associated with cellular stress and inflammation (Dreskin et al., 2001; Kyriakis and Avruch, 2001; Johnson and Lapadat, 2002). The two primary kinases that activate JNK are MKK4 and MKK7, while MKK3 and MKK6 phosphorylate p38. Activated JNK then phosphorylates and activates the transcription factor c-jun, which is involved in expression of a number of pro-inflammatory cytokine genes, 


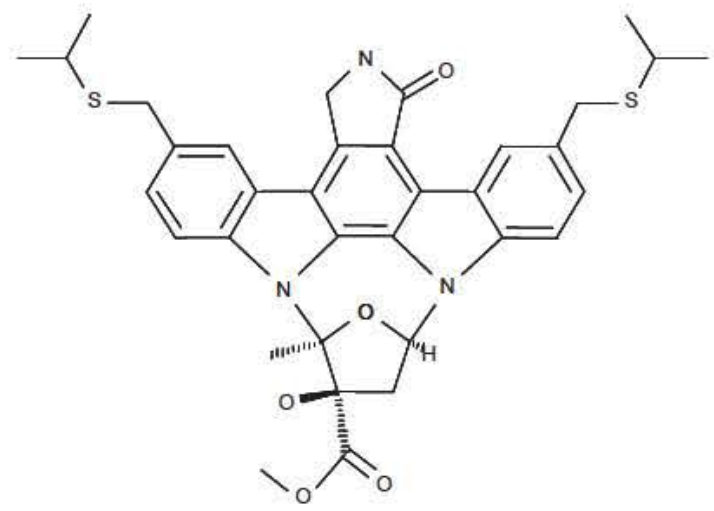

CEP-11004

Fig. 1. Chemical structure of CEP-11004. This isopropylthiomethyl derivative of the indolocarbazole $\mathrm{K} 252 \mathrm{a}$ is a highly potent MLK inhibitor (see text).

including tumor necrosis factor-alpha (TNF- $\alpha$ ) (Yao et al,, 1997). Gene and/or protein expression of TNF- $\alpha$ through the JNK pathway may be an important mechanism in sustaining an inflammatory response. Therefore, blockade of this pathway through JNK inhibition may reduce overexpression of TNF- $\alpha$.

Cells of monocyte/macrophage lineage are particularly responsive to lipopolysaccharide and have been shown to express and activate JNK in vitro (Dreskin et al., 2001), which can be reversed by treatment with CEP-11004 (Hidding et al., 2002). The downstream results of this acute response include activation of transcription factors that regulate immediate early genes, expression of adhesion molecules, and secretion of various pro-inflammatory cytokines including interleukin- 1 beta $(\mathrm{IL}-1 \beta)$ and TNF- $\alpha$ (Caroff et al., 2002; Yates et al., 2000; Liu et al., 2001). Thus, misregulation of, or chronic exposure to, TNF- $\alpha$ may exacerbate a pro-inflammatory response and lead to tissue damage and cell loss.

The current study evaluated the effects of CEP-11004 on lipopolysaccharide-mediated MAPK activation and TNF- $\alpha$ production in human monocytic and mouse microglial cell lines and in mice. These models were chosen to assess the ability of CEP-11004 to alter MAPK activation and TNF- $\alpha$ production during a biological stress response in vitro and in vivo. Phospho-JNK, phospho-p38, and TNF- $\alpha$ were increased after lipopolysaccharide treatment and the MLK inhibitor CEP-11004 inhibited these reactions, thereby providing a potential anti-inflammatory therapeutic.

\section{Materials and methods}

\subsection{Animals}

C57BL/6 mice were obtained from Charles River Laboratories (Wilmington, MA) and housed under con- trolled conditions and a 12-h day/night cycle. All studies conformed carefully to the guidelines outlined in the Guide for the Care and Use of Laboratory Animals from the U.S. Department of Health and Human Services and were approved by the Cephalon, Inc., Animal Care and Use Committee.

\subsection{Cells and reagents}

THP-1 cells were obtained from the American Type Culture Collection (Manassas, VA) and maintained in RPMI with $10 \%$ fetal bovine serum, $2 \mathrm{mM}$ L-glutamine, $1 \mathrm{mM}$ sodium pyruvate, $10 \mathrm{mM}$ HEPES, and $0.05 \mathrm{mM} 2$ mercaptoethanol. The murine microglia cell line BV-2 was kindly provided by E. Blasi (Perugia, Italy) and maintained in RPMI 1640 medium supplemented with $10 \%$ heatinactivated fetal calf serum, penicillin $(10,000 \mathrm{U} / \mathrm{ml})$, streptomycin $(10 \mathrm{mg} / \mathrm{ml})$, and glutamine $(2 \mathrm{mM})$.

Antibodies to JNK 1/3, phospho-JNK, and MKK4 were purchased from Santa Cruz Biotechnology (Santa Cruz, CA). Antibodies to p38, phospho-p38, and phospho-MKK4 were purchased from Cell Signaling Technology (Beverly, MA). Fluorescent goat anti-mouse Alexa Fluor-700 secondary antibody was from Molecular Probes (Eugene, OR) and goat anti-rabbit IRDye800 was from Rockland, Inc. (Gilbertsville, PA). Lipopolysaccharide was purchased from Sigma (St. Louis, MO). For THP-1 studies and in vivo studies, lipopolysaccharide was from Escherichia coli serotype O111:B4; for microglial experiments, lipopolysaccharide was from Salmonella abortus equi.

\subsection{Toxicity assay}

THP-1 cells were plated in 96-well culture dishes at $1.5 \times 10^{5}$ cells/well in a total volume of $200 \mu \mathrm{l}$. Cells were treated with various concentrations of lipopolysaccharide and CEP-11004 as indicated in figure legends. A CellTiter proliferation assay kit (Promega, Madison, WI) was used to test the viability of the cultures after treatment. This kit directly measured the conversion of MTS tetrazolium to a formazan product by metabolically active cells. A loss in signal denotes a loss of viability or increased toxicity.

\subsection{Cytokine enzyme linked immunosorbent assays (ELISAs)}

THP-1 cells were washed in phosphate-buffered saline (PBS) and resuspended in assay medium (RPMI, $2 \mathrm{mM}$ L-glutamine, and $0.05 \%$ bovine serum albumin) at a density of $1 \times 10^{6}$ cells $/ \mathrm{ml}$. Various concentrations of lipopolysaccharide and CEP-11004 were diluted in assay medium and applied in quadruplicate to a 96-well cell culture plate (Nunc, Rochester, NY). Cells were added at a density of $1 \times 10^{5}$ cells/well in a total volume of $200 \mu 1$ 
and incubated for various times. Samples were centrifuged at $200 \times g$ for $5 \mathrm{~min}$ and the supernatants were transferred to a new plate containing $1 \mu \mathrm{l} /$ well aprotinin (Sigma, St. Louis, MO) and frozen at $-80{ }^{\circ} \mathrm{C}$. TNF- $\alpha$ was measured using a standard human ELISA kit (R\&D Systems, Minneapolis, MN) according to the manufacturer's protocol. For BV-2 cells, TNF- $\alpha$ and interleukin-6 (IL-6) were measured in cell culture supernatants using mouse ELISA systems from eBioscience (San Diego, CA) according to the manufacturer's instructions.

\subsection{Real time polymerase chain reaction (PCR)}

Real-time PCR for TNF- $\alpha$ and MLKs in THP-1 cells was performed on a PRISM 7000 (Applied Biosystems, Foster City, CA) and analyzed with Sequence Detection System software. Specific primers were TNF- $\alpha$ sense: 5'-GACCCACGGCTTCACCCT-3', anti-sense: 5'TCCCGGATCATGCTTTCAG-3'; MLK1 sense: $5^{\prime}$ CAGCGGAGGAGGACCAAAA-3', anti-sense: 5' CGATTCAGCTTCCCAAACAC-3'; MLK2 sense: 5'-CCGTGTCTGCGAGGTTGTG-3', anti-sense: 5' 5C- $^{\prime}$ CACCGGGACCTCAAGTC-3'; MLK3 sense: 5'-GCCAAAGTCGGTGATCTTCAG-3', anti-sense: 5' CGTGATCTCAAGTCCAACAACATT-3'; GAPDH sense: 5'-GAAGGTGAAGGTCGGAGTCAAC-3', antisense: 5'-CAGAGTTAAAAGCAGCCCTGGT-3' . All primers were designed from GenBank sequences using Primer Express software and then custom-synthesized (Operon Technologies, Alameda, CA). Total RNA was isolated from lipopolysaccharide-treated THP-1 cells using a guanidinebased extraction reagent (TRI Reagent, Molecular Research Center, Cincinnati, OH). RNA was quantitated spectrophotometrically and $1.5 \mu \mathrm{g}$ was reverse-transcribed using a RetroScript cDNA synthesis kit (Ambion, Inc., Austin, TX). Synthesized cDNA was diluted to $10 \mathrm{ng} / \mathrm{ml}$ and $5 \mu \mathrm{l}$ was used in a $50 \mu \mathrm{l} \mathrm{PCR}$ reaction containing GAPDH-, TNF- $\alpha$, or MLKspecific primers and SYBR Green master mix (Applied Biosystems). The thermal profile was as follows: Stage 1: 1 repetition, $50{ }^{\circ} \mathrm{C}, 2 \mathrm{~min}$; Stage 2: 1 repetition, $95^{\circ} \mathrm{C}, 10 \mathrm{~min}$; Stage 3: 40 repetitions, $95^{\circ} \mathrm{C}, 15 \mathrm{~s} ; 60^{\circ} \mathrm{C}, 1 \mathrm{~min}$. MLK results are shown as average threshold cycle $\left(C_{\mathrm{T}}\right)$, which represents the PCR cycle at which an increase in fluorescence above a baseline signal can first be detected. Therefore, a lower threshold cycle corresponds to a higher expression value. TNF- $\alpha$ results are presented as a fold change compared to untreated controls so that comparisons could be made among different experiments. All values were normalized to a GAPDH internal standard.

BV-2 microglia were grown in $10 \mathrm{~cm}$ dishes and washed once with PBS, and total RNA was extracted using TRIzol reagent (Invitrogen, Carlsbad, CA) according to the manufacturer's protocol. RNA was DNase-1-treated using DNAfree $^{\mathrm{TM}}$ (Ambion, Huntingdon, UK) according to the manufacturer's protocol. Total RNA $(1 \mu \mathrm{g})$ was reverse-transcribed on a PTC-200 DNA Engine Thermal Cycler (VWR Interna- tional, Albertslund, Denmark) with TaqMan RT-Reagent (Applied Biosystems, Nærum, Denmark) using random hexamers. The cDNA was quantified using the SYBR Green PCR Master Mix kit (Applied Biosystems, Nærum, Denmark). PCR amplification was run in a 96-well experimental plate format on an iCycler Thermal Cycler equipped with an iCycler Optical System (Bio-Rad, Hercules, CA). The program set-up was $10 \mathrm{~min}$ at $95^{\circ} \mathrm{C}, 40$ cycles of $15 \mathrm{~min}$ at $95{ }^{\circ} \mathrm{C} / 1 \mathrm{~min}$ at $60{ }^{\circ} \mathrm{C}$. Quantification of $C_{\mathrm{T}}$ was performed using the iCycler data analysis software (Bio-Rad). The concentration of cDNA was calculated by comparing $C_{\mathrm{T}}$ of samples to $C_{\mathrm{T}}$ values of a standard curve obtained by a serial dilution of cDNA. Each sample was run in two reactions, one with the primer set of interest and one with a GAPDH primer set, and data are displayed as the ratio between the calculated starting concentration of the cDNA of interest and GAPDH. Primers used were GAPDH sense (accession no. NM 008084): 5'-TGCACCACCAACTGCTTA G-3', antisense: $5^{\prime}$-GGATGCAGGGATGATGTT C-3'. TNF- $\alpha$ sense (accession no. NM 0136935'-CTATGGCCCAGACCCTCACACTCA-3', anti-sense: 5'-CACTCCAGCTGCTCCTCCACTTG-3' ${ }^{\prime}$. Primers were designed using DNA star software package (DNASTAR Inc., Madison, WI).

\subsection{Western blot}

THP-1 cells were harvested by centrifugation and lysed in ice-cold FRAK buffer (1\% Triton X-100, $50 \mathrm{mM} \mathrm{NaCl}$, $30 \mu \mathrm{M}$ sodium pyrophosphate, $50 \mathrm{mM}$ sodium fluoride, 1 $\mathrm{mM}$ sodium vanadate, $10 \mathrm{mM}$ Tris $-\mathrm{HCl}, \mathrm{pH}$ 7.6). Lysates were sonicated and total protein was isolated by incubation on ice for $30 \mathrm{~min}$ followed by centrifugation at $16,000 \times \mathrm{g}$ at $4{ }^{\circ} \mathrm{C}$ for $20 \mathrm{~min}$. Total protein concentration in the supernatant was quantitated using a BCA assay kit (Pierce, Inc., Rockford, IL). Twenty-five or $50 \mu \mathrm{g}$ of protein was electrophoresed on 4-20\% SDS polyacrylamide NuPAGE gels using MES running buffer (Invitrogen) and transferred to polyvinylidene fluoride membrane. The membrane was blocked in LI-COR blocking buffer (LI-COR Biosciences, Lincoln, NE) for $1 \mathrm{~h}$ at room temperature followed by incubation with primary antibody at $4{ }^{\circ} \mathrm{C}$ overnight. Blots were incubated with a fluorescent-labeled secondary antibody for $1 \mathrm{~h}$ at room temperature. Proteins were visualized on an Odyssey Infrared imaging system (LI-COR) and quantitated using Odyssey software v1.0.

\subsection{Nitric oxide (NO) and prostaglandin $E_{2} \quad\left(P G E_{2}\right)$ production}

For measurement of NO production, nitrite was measured according to the Griess method. The supernatant of the cells was treated with $1 / 10(\mathrm{vol} / \mathrm{vol}) 1 \%$ sulfanilamide diluted in $1.2 \mathrm{M} \mathrm{HCl}$ and $1 / 10$ (vol/vol) $0.1 \% \mathrm{~N}$-naphthylethylenediamine. The absorption was measured after $5 \mathrm{~min}$ of incubation ( $\left.\lambda_{\text {signal }} 560-\lambda_{\text {background }} 690\right)$. For $\mathrm{PGE}_{2}$ production, cell supernatants were analyzed using a competitive 


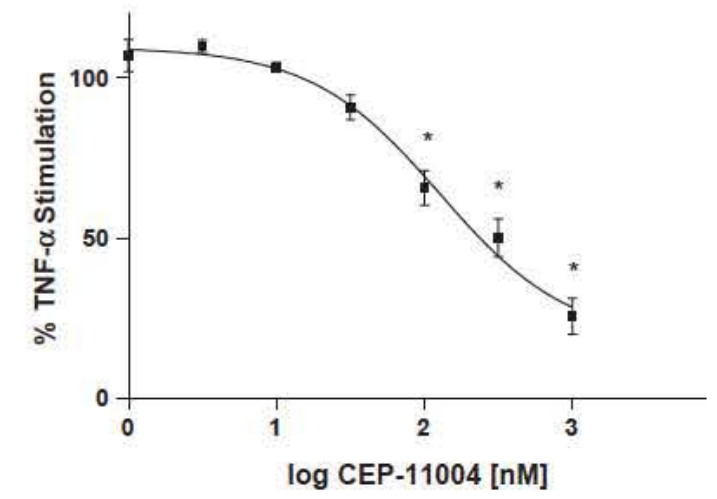

Fig. 2. CEP-11004 inhibited the lipopolysaccharide-induced increase in TNF- $\alpha$ from THP-1 human monocytes. THP-1 cells were incubated with 3 $\mu \mathrm{g} / \mathrm{ml}$ lipopolysaccharide with and without CEP-11004 (1 $1000 \mathrm{nM})$ for $6 \mathrm{~h}$. Cell supernatant was removed and assayed for the presence of TNF- $\alpha$. CEP11004 inhibited TNF- $\alpha$ release with an $\mathrm{IC}_{50}$ of $137.1 \pm 14.4 \mathrm{nM}(n \quad 4$ samples per group in three independent experiments). CEP-11004 at 1000 $\mathrm{nM}$ inhibited the lipopolysaccharide-induced response by $75 \%$. $* P<0.05$ compared to lipopolysaccharide alone.

enzyme immunoassay (Assay Designs, Inc., Ann Arbor, MI) according to the manufacturer's instructions.

\subsection{Phagocytosis assay}

Determination of macrophage phagocytosis was performed as described and modified previously (Uff et al., 1993; Lehner et al., 2002). Briefly, $3.0 \times 10^{4}$ microglia/ well were plated in 96-well microtiter plates (Cellstar, Greiner, Germany) and allowed to adhere for at least $3 \mathrm{~h}$. Then, tetramethylrhodamine-conjugated fluorescent $E$. coli particles (Molecular Probes, Leiden, The Netherlands) were sonicated and added to a final concentration of 10 $\mu \mathrm{g} / \mathrm{ml}$ at different time points. Phagocytosis was stopped by washing the cells twice with PBS to remove nonphagocytosed bacteria. Cells were then lysed by addition of $100 \mu \mathrm{l} /$ well of PBS with $20 \mathrm{mM}$ HEPES $+0.2 \%$ Triton $\mathrm{X}-100$. Fluorescence was determined at $530 \mathrm{~nm}$ excitation and $590 \mathrm{~nm}$ emission wavelengths using a fluorescence microplate reader (FL 600; Deelux Labortechnik, Goedenstorf, Germany). Cells without bacteria were used to determine the background fluorescence.

\subsection{Lipopolysaccharide mediated TNF $\alpha$ serum induction}

Male C57B1/6 mice, 6-8 weeks of age, were injected with an intraperitoneal (i.p.) dose of lipopolysaccharide at 12.5-20 $\mathrm{mg} / \mathrm{kg}$ and sacrificed at $2 \mathrm{~h}$ after injection by $\mathrm{CO}_{2}$ asphyxiation. CEP-11004 was administered subcutaneously (s.c.) $2 \mathrm{~h}$ prior to lipopolysaccharide injection at $0.1-10 \mathrm{mg} / \mathrm{kg}$ in $10 \%$ solutol. After sacrifice, animals were decapitated and blood was collected in $1.5 \mathrm{ml}$ microcentrifuge tubes. Blood samples were allowed to clot for $15 \mathrm{~min}$, and then centrifuged at $16,000 \times g$ for $15 \mathrm{~min}$ at $4{ }^{\circ} \mathrm{C}$. TNF- $\alpha$ was measured in the serum with a cytokine detection kit (Biosource International, Camarillo, CA) according to the manufacturer's protocol.

\subsection{Statistical analysis}

The mean \pm S.E.M. was determined for each treatment group in each experiment. Experiments indicated were normalized to untreated values for comparing multiple trials. Significance was defined by $P$ values $<0.05$ and determined by one-way analysis of variance (ANOVA) followed by Bonferroni's $t$ test for comparison of multiple groups with controls.

\section{Results}

3.1. Inhibition of TNF $\alpha$ secretion by CEP 11004 in lipopolysaccharide stimulated THP 1 human monocytes and mouse microglia

Fig. 2 shows that in THP-1 cells, CEP-11004 caused a concentration-dependent decrease in TNF- $\alpha$ secretion, with an $\mathrm{IC}_{50}$ of $137.1 \pm 14.4 \mathrm{nM}$. Background levels in untreated cells were undetectable, whereas a significant increase in TNF- $\alpha$ levels was observed in lipopolysaccharide-treated samples (data not shown). To verify that CEP-11004 was not toxic to the THP-1 cultures, an MTS toxicity assay was performed to determine the viability of cells after treatment with CEP-11004 for 3 or $24 \mathrm{~h}$. Concentrations of CEP11004 at 100 and $1000 \mathrm{nM}$ showed no significant decrease in cell viability compared to untreated controls (data not shown). To assess lipopolysaccharide toxicity, an MTS toxicity assay was performed to measure the viability of the cells after lipopolysaccharide treatment. Lipopolysaccharide did not cause significant loss of viability, including at $3 \mu \mathrm{g} /$ $\mathrm{ml}$ for $6 \mathrm{~h}$, which was the standard concentration in these studies (data not shown).

CEP-11004 also inhibited TNF- $\alpha$ release from microglial cells. Mouse BV-2 cells were treated for $4 \mathrm{~h}$ with various concentrations of lipopolysaccharide and CEP-11004, and TNF- $\alpha$ in cell culture supernatants was measured by ELISA.

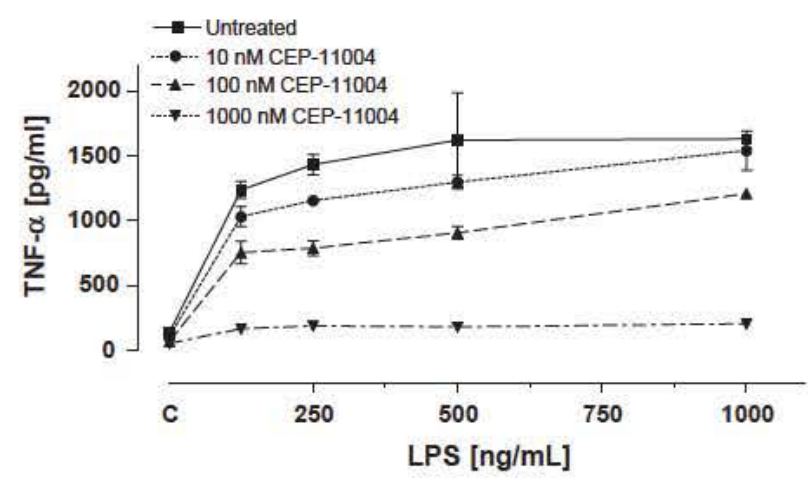

Fig. 3. CEP-11004 inhibited lipopolysaccharide-induced production of TNF- $\alpha$ from BV-2 mouse microglial cells. Cells were untreated or incubated with various concentrations of CEP-11004 for $4 \mathrm{~h}$ in the presence or absence (C) of lipopolysaccharide (LPS). Cell supernatant was removed and assayed for TNF- $\alpha$ by sandwich ELISA. Data are shown from triplicate determinations. 
Fig. 3 shows that addition of CEP-11004 resulted in a concentration-dependent decrease in TNF- $\alpha$ release from lipopolysaccharide-treated microglia, with $90 \%$ inhibition at $1000 \mathrm{nM}$ CEP-11004. Similar results were observed when CEP-11004 was administered up to $30 \mathrm{~min}$ after lipopolysaccharide treatment (data not shown).

\subsection{Effect of lipopolysaccharide and CEP 11004 on TNF $\alpha$ and MLK $m R N A$}

To determine if lipopolysaccharide and/or CEP-11004 influenced TNF- $\alpha$ mRNA expression in THP-1 cells, realtime PCR was performed with TNF- $\alpha$-specific primers (Fig. 4). Values are presented as fold change compared to untreated, so that comparisons could be made among different experiments. At $1 \mathrm{~h}$ after lipopolysaccharide treatment, TNF- $\alpha$ mRNA was elevated 25-fold compared to untreated control. Cotreatment with $300 \mathrm{nM}$ CEP11004 did not significantly decrease lipopolysaccharideinduced TNF- $\alpha$ mRNA levels, and CEP-11004 alone was not different from untreated controls. A 1-h pre-treatment with CEP-11004 produced similar results (data not shown).

The inability of CEP-11004 to affect TNF- $\alpha$ mRNA expression may indicate that MLKs are not expressed or not involved in regulating TNF- $\alpha$ gene expression. To test the first possibility, real-time PCR experiments were performed to measure MLK expression. Overall MLK levels were low but detectable, with MLK 3 levels the highest, followed by MLK 2, then MLK 1. Treatment with lipopolysaccharide and/or CEP-11004 had no effect on expression of MLKs in THP-1 cells (data not shown).

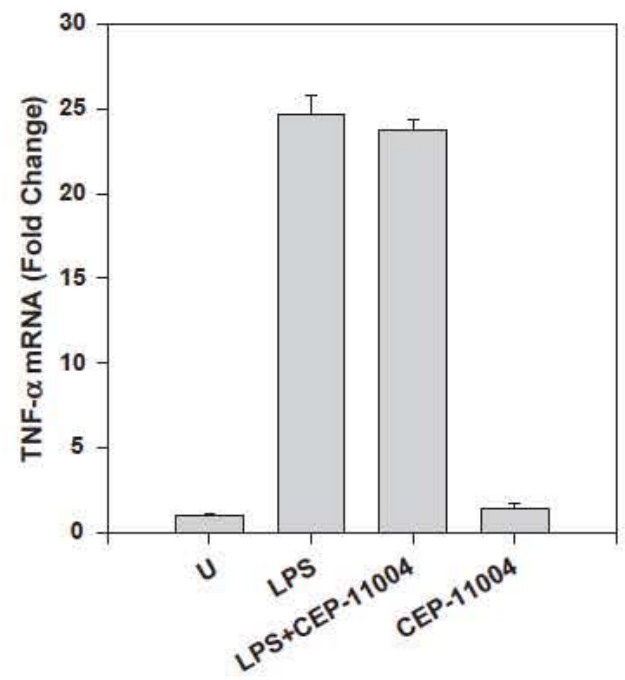

Fig. 4. Expression of TNF- $\alpha$ mRNA in lipopolysaccharide-treated THP-1 monocytes. Cells were untreated $(U)$ or stimulated for $1 \mathrm{~h}$ with $3 \mu \mathrm{g} / \mathrm{ml}$ lipopolysaccharide (LPS) with or without $300 \mathrm{nM} \mathrm{CEP-11004}$. Real-time PCR was performed to measure MLK and TNF- $\alpha$ gene expression. Six samples per group were examined in triplicate for all experiments. TNF- $\alpha$ mRNA was increased 25-fold after lipopolysaccharide treatment; however addition of 300 nM CEP-11004 did not significantly inhibit TNF- $\alpha$ mRNA levels.

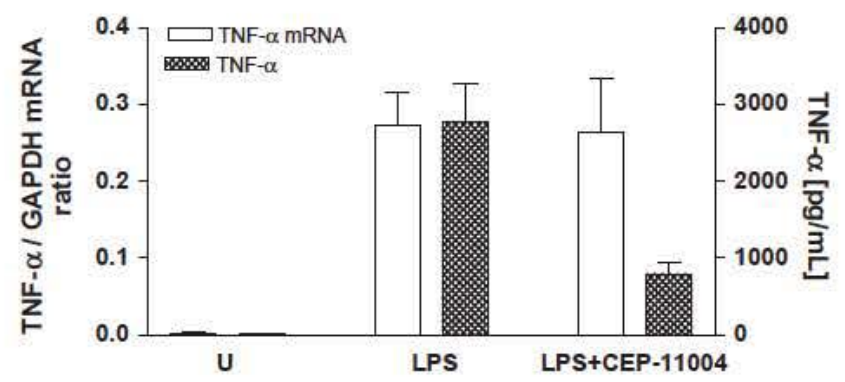

Fig. 5. Expression of TNF- $\alpha$ mRNA in lipopolysaccharide-treated BV-2 microglia. Cells were untreated (U) or stimulated for $2 \mathrm{~h}$ with $100 \mathrm{ng} / \mathrm{ml}$ lipopolysaccharide (LPS) with or without $300 \mathrm{nM} \mathrm{CEP-11004}$. TNF- $\alpha$ gene expression was measured by real-time PCR and normalized to GAPDH mRNA. Cell supematants were also collected for measurement of TNF- $\alpha$ secretion by ELISA. Lipopolysaccharide treatment caused a 54-fold increase in TNF- $\alpha$ mRNA and protein. Addition of CEP-11004 inhibited TNF- $\alpha$ protein secretion by $71 \%$, but had no effect on mRNA levels.

Also, in BV-2 cells incubated with $100 \mathrm{nM}$ lipopolysaccharide, CEP-11004 appeared to suppress TNF- $\alpha$ production without affecting the upregulation of TNF- $\alpha$ mRNA. The real-time PCR experiment in Fig. 5 shows that lipopolysaccharide treatment of BV-2 microglia caused

A
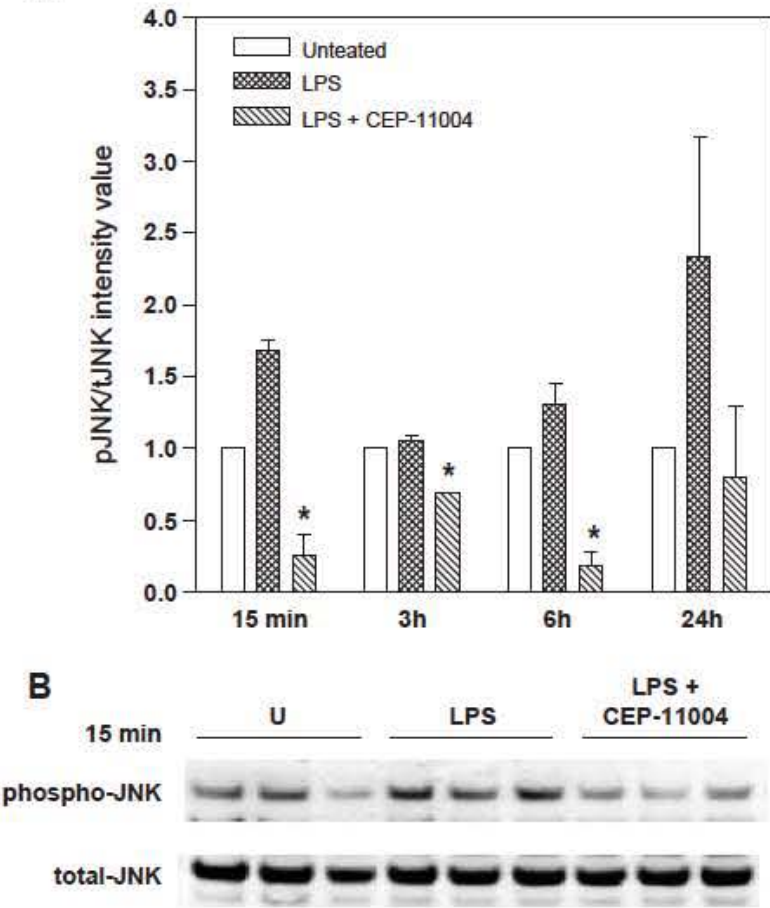

Fig. 6. Inhibition of JNK in lipopolysaccharide-stimulated THP-1 cells. THP-1 monocytes were stimulated for the indicated times with $3 \mu \mathrm{g} / \mathrm{ml}$ lipopolysaccharide (LPS) with or without $300 \mathrm{nM}$ CEP-11004. Immunoblotting was performed with antibodies recognizing phospho-p54 and p46 JNK, and total JNK. (A) Fluorescent intensity values were graphed as a ratio of phospho/total p54 JNK and normalized to untreated (U) values for each time point $(* P<0.05$ compared to lipopolysaccharide alone, $n \quad 6$ per group from three separate experiments). (B) Representative blot at the 15min time point showing the increase in phospho-p54 JNK with lipopolysaccharide treatment and inhibition of this response by CEP-11004. Total JNK levels remained stable in all treatment groups. 
A

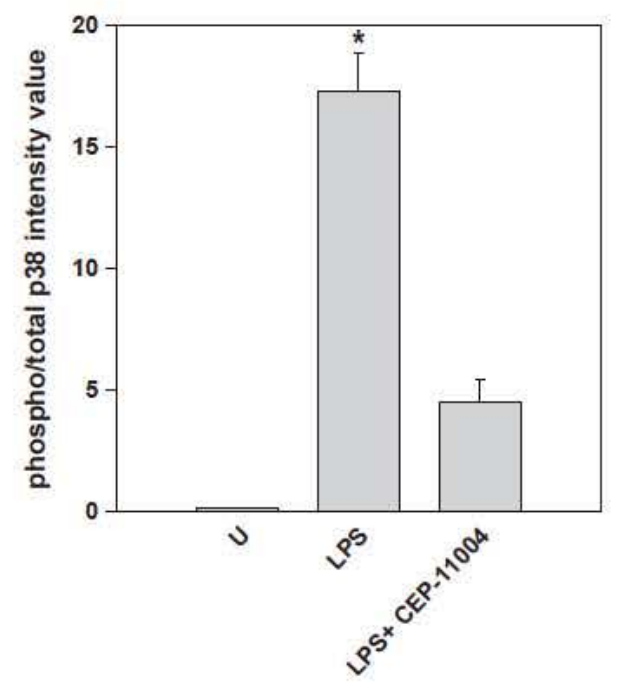

B

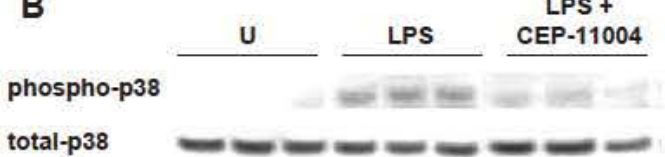

Fig. 7. Inhibition of p38 in lipopolysaccharide-stimulated THP-1 cells. THP-1 monocytes were stimulated for $3 \mathrm{~h}$ with $3 \mu \mathrm{g} / \mathrm{ml}$ lipopolysaccharide (LPS) with or without $300 \mathrm{nM}$ CEP-11004. Immunoblotting was performed with antibodies recognizing phospho-p38 and total-p38. (A) Fluorescent intensity values were graphed as a ratio of phospho/total p38 and normalized to untreated $(U)$ values $(* P<0.05$ compared to both untreated and lipopolysaccharide+CEP-11004, $n 7$ per group from three separate experiments). Lipopolysaccharide induced a marked activation of p38, which was inhibited by $74 \%$ in the presence of CEP-11004. (B) Representative blot showing an increase in phospho-p38 with lipopolysaccharide treatment and inhibition of this response by CEP-11004. Total p38 levels remained stable in all treatment groups.

a 54-fold increase in TNF- $\alpha$ mRNA within $2 \mathrm{~h}$, with no inhibition in the presence of CEP-11004. For comparison, TNF- $\alpha$ ELISA results from the same cells are also shown in Fig. 5 to indicate that in these same samples, secretion of TNF- $\alpha$ protein was inhibited by CEP-11004 treatment.

\subsection{CEP 11004 inhibits phosphorylation of JNK in lipo polysaccharide stimulated THP 1 cells}

To determine if activation of JNK occurred in this system, THP- 1 cells were stimulated with $3 \mu \mathrm{g} / \mathrm{ml}$ lipopolysaccharide with and without $300 \mathrm{nM} \mathrm{CEP}-11004$ for $15 \mathrm{~min}, 3 \mathrm{~h}, 6 \mathrm{~h}$, and $24 \mathrm{~h}$. Phosphorylation of 54 JNK, along with total JNK, was measured by Western blotting in three separate experiments. Values are presented as a ratio of phospho-p $54 \mathrm{JNK} /$ total JNK normalized to untreated values. Fig. $6 \mathrm{~A}$ shows that at $3 \mathrm{~h}$ after stimulation, lipopolysaccharide did not cause induction above controls; however, CEP-11004 inhibited JNK phosphorylation compared to untreated or lipopolysaccharide alone. At $15 \mathrm{~min}, 6 \mathrm{~h}$, and $24 \mathrm{~h}$ after treatment, lipopolysaccharide caused an increase in phosphorylation of JNK, which was inhibited by CEP-11004, even below basal levels. A representative blot from the 15-min time point (Fig. 6B) shows that phospho-JNK was highly regulated in this system, while total-JNK remained stable.

\subsection{CEP 11004 inhibits phosphorylation of p38 in lipo polysaccharide stimulated THP 1 human monocytes}

Besides JNK, another downstream target of MLK3 is the p38 MAPK. To determine if $\mathrm{p} 38$ is activated in this system, THP-1 cells were untreated or stimulated with $3 \mu \mathrm{g} / \mathrm{ml}$ lipopolysaccharide with and without $300 \mathrm{nM}$ CEP-11004 for $3 \mathrm{~h}$. Phosphorylation of p38, along with total p38, was measured by Western blot in three separate experiments using antibodies to phospho-p38 and total-p38 (Fig. 7). Values are presented as a ratio of phospho-p38/total-p38 normalized to untreated values (Fig. 7A). Lipopolysaccharide stimulated a 17-fold increase in p38 activation above untreated cells, which was inhibited at $74 \%$ with $300 \mathrm{nM} \mathrm{CEP}-11004$. A representative blot (Fig. 7B) shows the increase in phosphop38 and the inhibition by CEP-11004. Total p38 remained stable in all treatments.

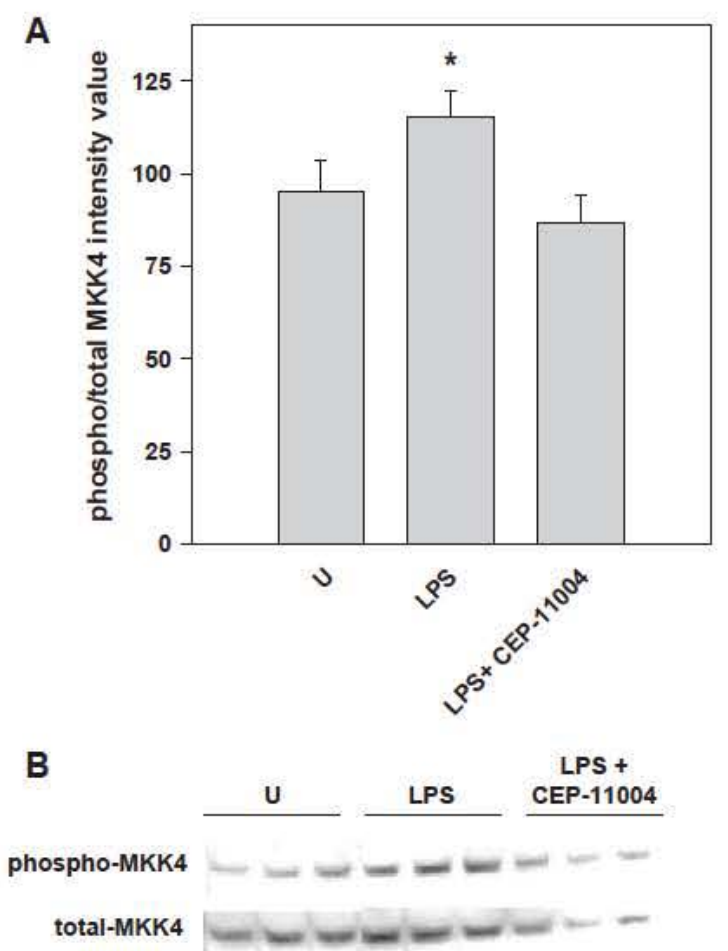

Fig. 8. Inhibition of MKK 4 in lipopolysaccharide-treated THP-1 monocytes. Cells were stimulated for $3 \mathrm{~h}$ with $3 \mu \mathrm{g} / \mathrm{ml}$ lipopolysaccharide (LPS) with or without $300 \mathrm{nM}$ CEP-11004. Immunoblot was performed with antibodies recognizing phospho-MKK4 and total-MKK4. Fluorescent intensity values were graphed as a ratio of phospho/total MKK4 and normalized to untreated (U) values for each time point $\left({ }^{*} P<0.05\right.$ compared to untreated and lipopolysaccharide+CEP-11004, n 67 per group from three separate experiments). Lipopolysaccharide caused an increase in phospho-MKK4, which was prevented by CEP-11004. (B) Representative blot showing an increase in phospho-MKK4 with lipopolysaccharide treatment and inhibition of this response by CEP-11004. Total MKK4 levels tended to mimic phospho-MKK4, particularly in the CEP-11004 group. 

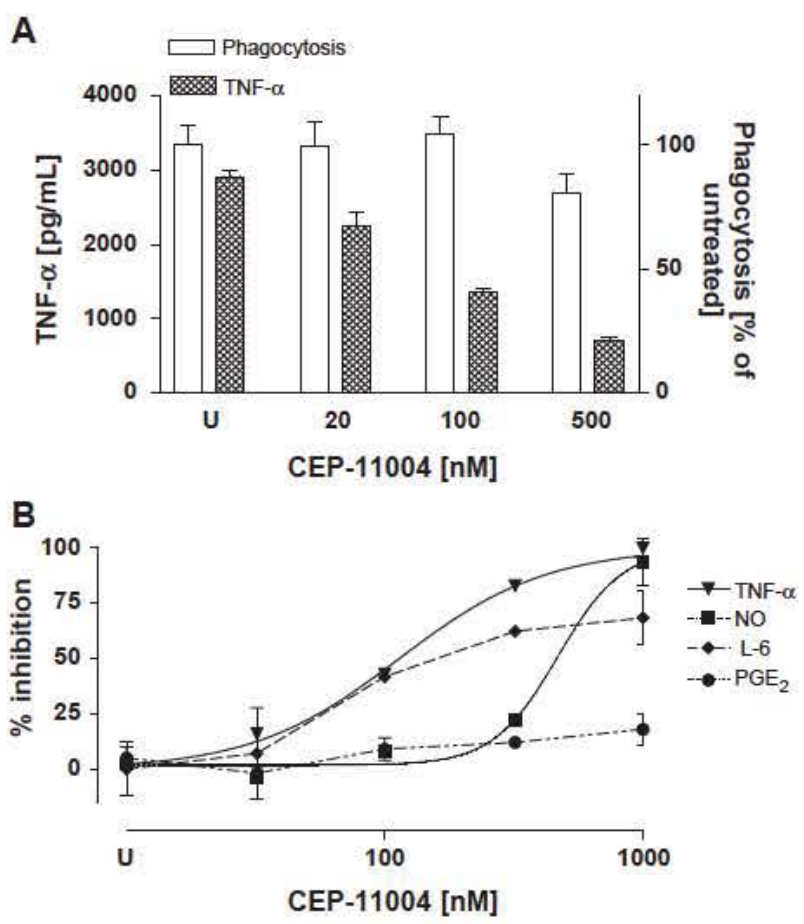

Fig. 9. Selective inhibition of cytokine secretion by CEP-11004. (A) Cells were untreated $(U)$ or treated with the indicated concentrations of CEP-11004 for $45 \mathrm{~min}$, followed by a $4 \mathrm{~h}$ incubation with fluorescent $E$. coli bacteria. Phagocytosis was determined by measuring the number of fluorescent bacteria within $\mathrm{BV}-2$ cells fluorimetrically. TNF- $\alpha$ (triggered by $E$. coli) was measured from the same cultures. (B) BV-2 cells were untreated (U) or incubated with $100 \mathrm{ng} / \mathrm{ml}$ lipopolysaccharide and various concentrations of CEP-11004. TNF- $\alpha$, IL-6, PGE $_{2}$, and NO measurements were made from the same culture supernatants. Data are from quadruplicate experiments.

\subsection{Effect of lipopolysaccharide and CEP 11004 on MKK4 activation}

MKK4 is a dual-specificity kinase that can be activated by MLKs and can subsequently phosphorylate JNK. To measure MKK4 activation, THP-1 cells were stimulated with $3 \mu \mathrm{g} / \mathrm{ml}$ lipopolysaccharide with and without $300 \mathrm{nM} \mathrm{CEP}-11004$ for $3 \mathrm{~h}$. Phospho-MKK 4 and total-MKK 4 were measured by Western blot and results are presented as a ratio of phosphoMKK4/total-MKK4 normalized to untreated values (Fig. 8). Lipopolysaccharide caused a significant and reproducible $17 \%$ activation of MKK 4 above untreated cells, which was completely inhibited by $300 \mathrm{nM}$ CEP-11004. A representative blot (Fig. 8B) shows the increase in phospho-MKK4 and the inhibition by CEP-11004. Total-MKK4 tended to follow the same expression pattern as phospho-MKK4.

\subsection{Selective modulation of inflammation by CEP 11004}

Reactive microglia display functional characteristics such as induction of phagocytosis, prostaglandin synthesis, and nitric oxide (NO) production. To determine if CEP-11004 influences all of these parameters similarly, BV-2 microglia were incubated with lipopolysaccharide and CEP-11004 and assessed for various endpoints. Although CEP-11004 inhibited TNF- $\alpha$ secretion by $75 \%$, it had little effect on phagocytosis, a function of macrophages important in resolving damage (Fig. 9A). The effect of CEP-11004 on secretion of the inflammatory mediators $\mathrm{NO}$, IL-6, prostaglandin $\mathrm{E}_{2}\left(\mathrm{PGE}_{2}\right)$, and TNF- $\alpha$ was directly compared within the same cultures (Fig. 9B). While inhibition of TNF- $\alpha$ and IL- 6 secretion was seen at 100 $\mathrm{nM}$ CEP-11004, NO production was not inhibited at all at this concentration. Only at $1000 \mathrm{nM}$ did CEP-11004 inhibit NO production, whereas $\mathrm{PGE}_{2}$ production was not significantly inhibited at any concentration.

\subsection{CEP 11004 inhibited the in vivo lipopolysaccharide induced TNF $\alpha$ increase}

The effect of in vivo lipopolysaccharide administration on serum TNF- $\alpha$ levels in C57B1/6 mice was examined. An elevation of TNF- $\alpha$ in serum was observed in samples from

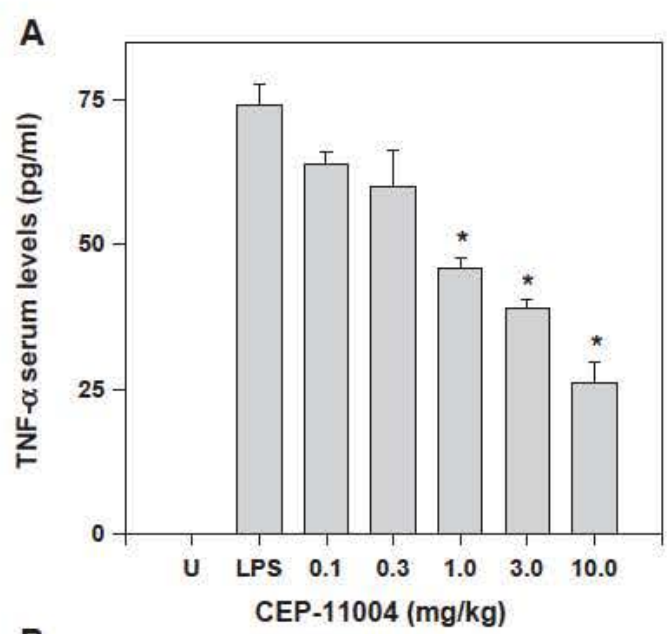

B

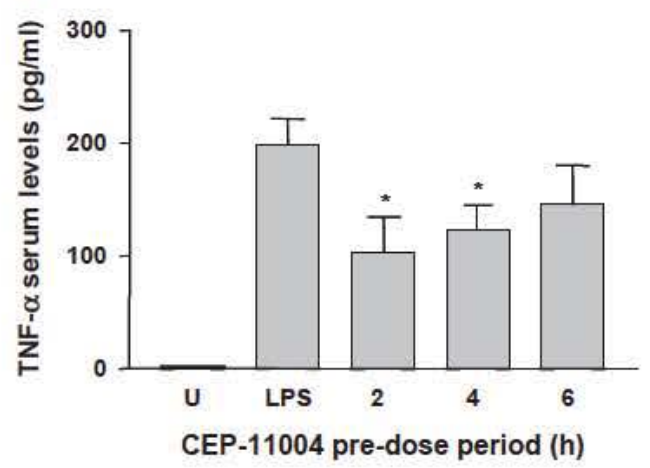

Fig. 10. CEP-11004 inhibited the lipopolysaccharide induced increase in TNF- $\alpha$ from mouse serum. (A) C57BV/ 6 mice were untreated or injected with $20 \mathrm{mg} / \mathrm{kg}$ lipopolysaccharide (LPS) and various concentrations of CEP11004 as described in Materials and methods ( $n 3$ per group). CEP-11004 significantly inhibited the lipopolysaccharide-induced response $\left({ }^{*} P<0.05\right.$ compared to lipopolysaccharide alone). (B) Pre-treatment with $10 \mathrm{mg} / \mathrm{kg}$ CEP-11004 at 2 or $4 \mathrm{~h}$ prior to lipopolysaccharide injection significantly inhibited the TNF- $\alpha$ serum elevation $\left({ }^{*} P<0.05\right.$ compared to lipopolysaccharide alone). A $6 \mathrm{~h}$ pre-treatment caused a $27 \%$ inhibition, but was not a statistically significant alteration of TNF- $\alpha$ levels ( $n \quad 3$ per group). 
all animals injected with lipopolysaccharide, with maximal levels of $1251 \mathrm{pg} / \mathrm{ml}$ achieved at a dose of $50 \mathrm{mg} / \mathrm{kg}$ (data not shown). Subsequent experiments used a dose of 20 $\mathrm{mg} / \mathrm{kg}$. Fig. 10A demonstrates a dose-dependent inhibition of TNF- $\alpha$ serum levels in mice treated $2 \mathrm{~h}$ prior to lipopolysaccharide administration with $0.1-10 \mathrm{mg} / \mathrm{kg}$ CEP-11004. Inhibition of $38 \%$ was seen with $1 \mathrm{mg} / \mathrm{kg}$ CEP-11004, 47\% with $3 \mathrm{mg} / \mathrm{kg}$, and $65 \%$ with $10 \mathrm{mg} / \mathrm{kg}$. TNF- $\alpha$ levels were not detectable in untreated animals. Pre-treatment with CEP-11004 at 2, 4, and $6 \mathrm{~h}$ prior to lipopolysaccharide injection showed $48 \%$ and $39 \%$ inhibition of TNF- $\alpha$ at 2 and $4 \mathrm{~h}$, respectively, but did not reach significance at $27 \%$ inhibition for $6 \mathrm{~h}$ pretreatment (Fig. 10B).

\section{Discussion}

This report demonstrates that CEP-11004 is a potent inhibitor of TNF- $\alpha$ production both in vitro and in vivo. The drug acts by inhibiting lipopolysaccharide-mediated activation of MAPK signaling pathways. Although lipopolysaccharide-induced TNF- $\alpha$ secretion is inhibited by CEP11004 , early gene expression is not altered, suggesting that the attenuation in secretion is not attributable to inhibition of mRNA expression. This indicates that different signaling pathways can be utilized for these processes. Indeed, it seems that the lipopolysaccharide signaling cascade leading to overall TNF- $\alpha$ secretion diverges and separately affects different steps in the control of transcription and translation. Swantek et al. (1997) reported that transcription is primarily controlled through $\mathrm{NF}-\kappa \mathrm{B}$, whereas translation is dependent on a translational repression sequence in the $3^{\prime}$ untranslated region of the TNF- $\alpha$ gene. The data presented suggest that CEP-11004 inhibits TNF- $\alpha$ production through a posttranscriptional mechanism, but does not interfere with initial transcriptional signaling. It is possible, however, that CEP11004 may have transcriptional effects at later time points after lipopolysaccharide treatment. In support of this, a CEP-11004-mediated decrease in TNF- $\alpha$ mRNA levels was observed from 8 to $24 \mathrm{~h}$ after lipopolysaccharide treatment in BV-2 cells (data not shown). This could indicate that multiple time-dependent mechanisms are utilized for regulation of $\mathrm{TNF}-\alpha$ transcription during an inflammatory response.

In agreement with previous findings (Dreskin et al., 2001; Nakajima et al., 2004), phosphorylation of p54 JNK and p38 was induced in lipopolysaccharide-treated THP-1 monocytes and microglia. The current studies showed further that this JNK activation is inhibited by CEP11004. These data suggest that CEP-11004 may influence JNK- and p38-dependent inflammatory responses through inhibition of these signaling pathways. This may occur through various mechanisms, including inhibition of TNF- $\alpha$ secretion by phosphatase-mediated deactivation of JNK (Matsuguchi et al., 2001) or inhibition of p-38 (Nakajima et al., 2004), and activation of transcription factors that regulate TNF- $\alpha$ expression (Yao et al., 1997). However, these mechanisms may not be utilized in all cellular responses, since treatment with CEP-11004 did not affect phagocytosis, which is required for antigen clearance, or $\mathrm{PGE}_{2}$ production, which can down-regulate NO production in macrophages (D'Acquisto et al., 1998).

Activation of JNK can occur via phosphorylation by the upstream kinases MKK4 and MKK7, which are activated by MLKs. Activity of the MLKs can be regulated through interactions with JNK-interacting proteins (JIPs). JIPs are scaffolding proteins that play a role in the assembly and function of various kinase complexes in the JNK pathway (Gallo and Johnson, 2002). The present study indicates that MKK4 is activated $3 \mathrm{~h}$ after lipopolysaccharide treatment in THP-1 cells. However, MKK4 phosphorylation, although reproducible, was increased by only $17 \%$. Therefore, CEP-11004 had a strong inhibitory effect on lipopolysaccharide-induced JNK phosphorylation that may be only partially mediated through inhibition of the MKK4 pathway. It is possible that MKK7 may also be utilized for signaling. This is supported by studies suggesting that different cell signals can mediate specific interactions between MLKs and MKKs and that the combination of activated kinases in a particular pathway is stimulationand cell-dependent and may involve multiple pathways (Chen et al., 2002; Gallo and Johnson, 2002; Shen et al., 2003). Moreover, it is known that JIP1 binds MKK7 but not MKK4, which may indicate a preferential substrate in JIP1-mediated signaling (Yasuda et al., 1999). At present, it is not known which JIPs are most active in THP-1 and $\mathrm{BV}-2$ cell systems.

TNF- $\alpha$ production is seen in many inflammatory disorders and may contribute to secondary damage that further worsens a disease state (Tracey and Cerami, 1994). For example, in Alzheimer's disease, upregulation of TNF$\alpha$ can be induced by $A \beta$ peptides and exacerbate a neurotoxic environment (Yates et al., 2000; Liu et al., 2001). In autoimmune disorders such as multiple sclerosis, production of TNF- $\alpha$ and other pro-inflammatory cytokines can play a role in demyelination and cell loss (Keegan and Noseworthy, 2002). The present studies in lipopolysaccharide-treated mice indicate that MLK inhibition with CEP11004 can effectively decrease circulating TNF- $\alpha$ levels. Therefore, CEP-11004 and similar compounds may be therapeutic in a number of diseases by decreasing TNF- $\alpha$ and possibly other inflammatory mediators.

\section{Acknowledgments}

The authors wish to thank Tom Connors, Beth Ann McKenna, Andreas Rassow, and Dr. Joanne Mathiasen for technical expertise in characterizing CEP-11004 and establishing in vitro assays, and Beth Ann Thomas and Jeff Thomas for assistance with real-time PCR techniques. 


\section{References}

Caroff, M., Karibian, D., Cavaillon, J.M., Haeffner-Cavaillon, N., 2002. Structural and functional analyses of bacterial lipopolysaccharides. Microbes Infect. 4, 915926.

Chen, W., White, M.A., Cobb, M.H., 2002. Stimulus-specific requirements for MAP3 kinases in activating the JNK pathway. J. Biol. Chem. 277, 4910549110.

D’Acquisto, F., Sautebin, L., Iuvone, T., Di Rosa, M., Carnuccio, R., 1998. Prostaglandins prevent inducible nitric oxide synthase protein expression by inhibiting nuclear factor-kappaB activation in J774 macrophages. FEBS Lett. 440, 7680.

Dreskin, S.C., Thomas, G.W., Dale, S.N., Heasley, L.E., 2001. Isoforms of Jun kinase are differentially expressed and activated in human monocyte/macrophage (THP-1) cells. J. Immunol. 166, 56465653.

Gallo, K.A., Johnson, G.L., 2002. Mixed-lineage kinase control of JNK and p38 MAPK pathways. Nat. Rev., Mol. Cell Biol. 3, 663672.

Hidding, U., Mielke, K., Waetzig, V., Brecht, S., Hanisch, U., Behrens, A., Wagner, E., Herdegen, T., 2002. The c-Jun N-terminal kinases in cerebral microglia: immunological functions in the brain. Biochem. Pharmacol. 64, 781788 .

Johnson, G.L., Lapadat, R., 2002. Mitogen-activated protein kinase pathways mediated by ERK, JNK, and p38 protein kinases. Science 298, 19111912.

Keegan, B.M., Noseworthy, J.H., 2002. Multiple sclerosis. Annu. Rev. Med. 53, 285302.

Kyriakis, J.M., Avruch, J., 2001. Mammalian mitogen-activated protein kinase signal transduction pathways activated by stress and inflammation. Physiol. Rev. 81, 807869.

Lehner, M.D., Schwoebel, F., Kotlyarov, A., Leist, M., Gaestel, M., Hartung, T., 2002. MAPKAP kinase 2 deficient mice show increased susceptibility to Listeria monocytogenes infection. J. Immunol. 168, 46674673.

Liu, B., Wang, K., Gao, H.M., Mandavilli, B., Wang, J.Y., Hong, J.S., 2001. Molecular consequences of activated microglia in the brain, overactivation induces apoptosis. J. Neurochem. 77, 182189.

Matsuguchi, T., Musikacharoen, T., Johnson, T.R., Kraft, A.S., Yoshikai, Y., 2001. A novel mitogen-activated protein kinase phosphatase is an important negative regulator of lipopolysaccharide-mediated c-Jun N- terminal kinase activation in mouse macrophage cell lines. Mol. Cell. Biol. 21, 69997009 .

Murakata, C., Kaneko, M., Gessner, G., Angeles, T.S., Ator, M.A., O’Kane, T.M., McKenna, B.A., Thomas, B.A., Mathiasen, J.R., Saporito, M.S., Bozyczko-Coyne, D., Hudkins, R.L., 2002. Mixed lineage kinase activity of indolocarbazole analogues. Bioorg. Med. Chem. Lett. 12, 147150 .

Nakajima, K., Tohyama, Y., Kohsaka, S., Kurihara, T., 2004. Protein kinase $\mathrm{C}$ alpha requirement in the activation of p38 mitogen-activated protein kinase, which is linked to the induction of tumor necrosis factor alpha in lipopolysaccharide-stimulated microglia. Neurochem. Int. 44, 205214.

Shen, Y.H., Godlewski, J., Zhu, J., Sathyanarayana, P., Leaner, V., Birrer, M.J., Rana, A., Tzivion, G., 2003. Cross-talk between JNK/SAPK and ERK/MAPK pathways. J. Biol. Chem. 278, 2671526721.

Swantek, J.L., Cobb, M.H., Geppert, T.D., 1997. Jun N-terminal kinase/stress-activated protein kinase (JNK/SAPK) is required for lipopolysaccharide stimulation of tumor necrosis factor alpha (TNF-alpha) translation, glucocorticoids inhibit TNF-alpha translation by blocking JNK/SAPK. Mol. Cell. Biol. 17, 62746282.

Tracey, K.J., Cerami, A., 1994. Tumor necrosis factor, a pleiotropic cytokine and therapeutic target. Annu. Rev. Med. 45, 491503.

Uff, C.R., Pockley, A.G., Phillips, R.K., 1993. A rapid microplate-based fluorometric assay for phagocytosis. Immunol. Invest. 22, 407413.

Wang, L.H., Paden, A.J., Johnson Jr., E.M., 2005. Mixed-lineage kinase inhibitors require the activation of Trk receptors to maintain longterm neuronal trophism and survival. J. Pharmacol. Exp. Ther. 312, 10071019.

Yao, J., Mackman, N., Edgington, T.S., Fan, S.T., 1997. Lipopolysaccharide induction of the tumor necrosis factor-alpha promoter in human monocytic cells. Regulation by Egr-1, c-Jun, and NF-kappaB transcription factors. J. Biol. Chem. 272, 1779517801.

Yasuda, J., Whitmarsh, A.J., Cavanagh, J., Sharma, M., Davis, R.J., 1999. The JIP group of mitogen-activated protein kinase scaffold proteins. Mol. Cell. Biol. 19, 72457254.

Yates, S.L., Burgess, L.H., Kocsis-Angle, J., Antal, J.M., Dority, M.D., Embury, P.B., Piotrkowski, A.M., Brunden, K.R., 2000. Amyloid beta and amylin fibrils induce increases in proinflammatory cytokine and chemokine production by THP-1 cells and murine microglia. J. Neurochem. 74, 10171025. 